\title{
RELACIÓN ENTRE NIVELES DE TESTOSTERONA EN SUERO Y CÁNCER PROSTÁTICO
}

\author{
P. RIVERA G., R. TAGLE A., S. MIR C., R. GONZÁLEZ I. \\ Unidad de Urología. Facultad de Medicina. Universidad de La Frontera. Temuco. Chile.
}

Actas Urol Esp. 27 (10): 788-792, 2003

\section{RESUMEN}

RELACIÓN ENTRE NIVELES DE TESTOSTERONA EN SUERO Y CÁNCER PROSTÁTICO

INTRODUCCIÓN: La asociación entre testosterona y cáncer prostático es mal comprendida. Los niveles de testosterona no son concluyentes para distinguir procesos prostáticos benignos de malignos.

El objetivo de nuestro trabajo es determinar la relación existente entre niveles de testosterona en suero y cáncer prostático.

MATERIAL Y MÉTODO: Se estudiaron en forma prospectiva 38 pacientes portadores de cáncer prostático y un grupo control de 32, con edades que fluctuaron entre los 50 y 80 años. La testosterona total y libre fue determinada por radioinmunoensayo. El análisis estadístico realizado fue descriptivo inferencial, con bandas de confianza de 95\%.

RESULTADOS: Los valores promedio de testosterona total y libre, fueron significativamente menores en los pacientes portadores de cáncer. No se demostró ninguna correlación entre gleason, antígeno prostático y etapa tumoral con testosterona sérica.

CONCLUSIONES: Nuestro trabajo demostró que la testosterona es significativamente menor en los pacientes con cáncer prostático en relación a los controles.

PALABRAS CLAVE: Cáncer prostático. Testosterona.

\section{ABSTRACT}

RELATION BETWEEN LEVELS OF TESTOSTERONE IN SERUM AND PROSTATE CANCER

INTRODUCTION: The association between testosterone and prostate cancer badly is understood. The testosterone levels are not conclusive to distinguish benign prostate processes of malignant. The objective of our work is to determine the relation between levels of serum testosterone and prostate cancer.

MATERIAL AND METHOD: 38 patients with prostate cancer and a control group of 32 were studied in prospective form, with ages that fluctuated between the 50 and 80 years. The total and free testosterone was determined by radioinmunoensayo. The statistical analysis was descriptive inferential, with bands of $95 \%$ confidence.

RESULTS: The average values of total and free testosterone, were significantly smaller in patients with prostate cancer. Any correlation between gleason, prostate antigen and stage with testosterone, was not demonstrated.

CONCLUSIONS: Our work demonstrated that the testosterone is significantly smaller in the patients with prostate cancer that in the controls.

KEY WORDS: Prostate cancer. Testosterone. 
$\mathrm{L}^{\circ}$ os andrógenos endógenos incluyendo la testosterona y la dihidrotestosterona (DHT) son los responsables del normal crecimiento y desarrollo de los órganos sexuales y mantención de las características sexuales secundarias.

Este efecto incluye la maduración y crecimiento de la próstata, vesículas seminales, pene y escroto; el desarrollo de la distribución pilosa, engrosamiento de la laringe, musculatura corporal y distribución de grasa.

En el hombre la testosterona en el suero se encuentra distribuida en tres formas: unida firmemente a la globulina sexual, unida a la albúmina y en forma libre, sólo estas dos últimas son biodisponibles.

La asociación entre testosterona y cáncer prostático es mal comprendida. Los niveles de testosterona no son concluyentes para distinguir procesos prostáticos benignos de malignos ${ }^{1}$.

Existe un trabajo que relaciona la disminución de testosterona total y libre, con pacientes portadores de cáncer prostático, en los cuales, el examen rectal y antígeno prostático son norma$\operatorname{les}^{2}$. Además hay otro en que relaciona la disminución de testosterona libre y la mayor agresividad del cáncer prostático ${ }^{3}$.

El objetivo de nuestro trabajo es determinar la relación existente entre niveles de testosterona en suero y cáncer prostático.

\section{MATERIAL Y MÉTODO}

Se estudiaron en forma prospectiva 38 pacientes portadores de cáncer prostático y un grupo control de 32 , con edades que fluctuaron entre los 50 y 80 años.

Se realizaron biopsias prostáticas a los pacientes con tacto rectal sospechoso y o antígeno prostático alterado. Luego de demostrar el cáncer, se etapificaron y se les tomó muestra de sangre para medir testosterona total y libre, teniendo el cuidado de ser tomadas en la mañana entre las 8:30 y 9:30 h, lo mismo al grupo control.

La testosterona total se determinó por radioinmunoensayo (Immunotech) y los valores mínimos y máximos fueron 3 y $12 \mathrm{ng} / \mathrm{ml}$, respectivamente.

La testosterona libre también fue determinada por radioinmunoensayo con valores normales de 4,5 y $23 \mathrm{pg} / \mathrm{ml}$.
Además se evaluaron con Antígeno Prostático Específico (APE), Gleason y se etapificaron.

$\mathrm{El}$ análisis estadístico realizado fue descriptivo inferencial, con bandas de confianza de 95\% para determinar los valores, donde las diferencias promedios de cada grupo se hacen significativas. Para todos los análisis fue utilizado el software estadístico STATA 6.0

\section{RESULTADOS}

La edad promedio de los pacientes portadores de cáncer prostático fue de 69,9 años (54-94) y la del grupo control 65,6 (50-84), no existiendo diferencia estadísticamente significativa entre ambos grupos $(\mathrm{P}=0,06)$ (Tabla $\mathrm{I})$.

La relación entre testosterona y grupo etario, demostró que si bien es cierto, ésta disminuye a medida que aumenta la edad (como se observa en los pacientes controles); los pacientes portadores de cáncer tienen valores inferiores a éstos (Tablas II y III).

TABLA I

DISTRIBUCIÓN PACIENTES POR GRUPO ETARIO

\begin{tabular}{|c|c|c|}
\hline Grupo Etario & Cáncer & Control \\
\hline $50-59$ & $4(10 \%)$ & $8(25 \%)$ \\
\hline $60-69$ & $15(39 \%)$ & $11(34 \%)$ \\
\hline $70-79$ & $12(32 \%)$ & $10(31 \%)$ \\
\hline$>80$ & $7(19 \%)$ & $3(10 \%)$ \\
\hline Total & $\mathbf{3 8}(\mathbf{1 0 0} \%)$ & $\mathbf{3 2}(\mathbf{1 0 0} \%)$ \\
\hline
\end{tabular}

TABLA II

NIVELES DE TESTOSTERONA TOTAL POR GRUPO ETARIO

\begin{tabular}{|c|c|c|}
\hline \multirow{2}{*}{ Grupo Etario } & \multicolumn{2}{|c|}{ Testosterona total (ng/ ml) } \\
\cline { 2 - 3 } & Cáncer & Control \\
\hline $50-59$ & 1,98 & 4,05 \\
\hline $60-69$ & 2,38 & 3,48 \\
\hline $70-79$ & 2,54 & 2,66 \\
\hline$>80$ & 2,30 & 3,15 \\
\hline
\end{tabular}

TABLA III

NIVELES DE TESTOSTERONA LIBRE POR GRUPO ETARIO

\begin{tabular}{|c|c|c|}
\hline \multirow{2}{*}{ Grupo Etario } & \multicolumn{2}{|c|}{ Testosterona total (ng/ ml) } \\
\cline { 2 - 3 } & Cáncer & Control \\
\hline $50-59$ & 8,31 & 15,83 \\
\hline $60-69$ & 10,65 & 13,83 \\
\hline $70-79$ & 10,51 & 12,82 \\
\hline$>80$ & 12,6 & 11,94 \\
\hline
\end{tabular}


Considerando los niveles promedio de testosterona total y libre, éstos fueron significativamente menor en los pacientes portadores de cáncer, en relación al grupo control (Tabla IV) (Gráficos 1 y 2).

Un $46 \%$ de los pacientes portadores de cáncer prostático tenían Gleason 7 y un $27 \%$ Gleason 8. No se demostró ninguna correlación entre Gleason y testosterona libre (Tabla V).

\section{TABLA IV}

VALORES PROMEDIO DE TESTOSTERONA TOTAL Y LIBRE

\begin{tabular}{||l|c|c|l||}
\hline Grupo Estudio & Cáncer & Control & Valor $\mathbf{p}$ \\
\hline $\begin{array}{l}\text { Testosterona total } \\
\text { (ng/ml) }\end{array}$ & 2,33 & 3,33 & $\begin{array}{l}\text { 0,003 significativo, } \\
\text { grupos distintos }\end{array}$ \\
\hline $\begin{array}{l}\text { Testosterona libre } \\
\text { (pg/ml) }\end{array}$ & 10,04 & 13,83 & $\begin{array}{l}\text { 0,0004 significativo, } \\
\text { grupos distintos }\end{array}$ \\
\hline
\end{tabular}

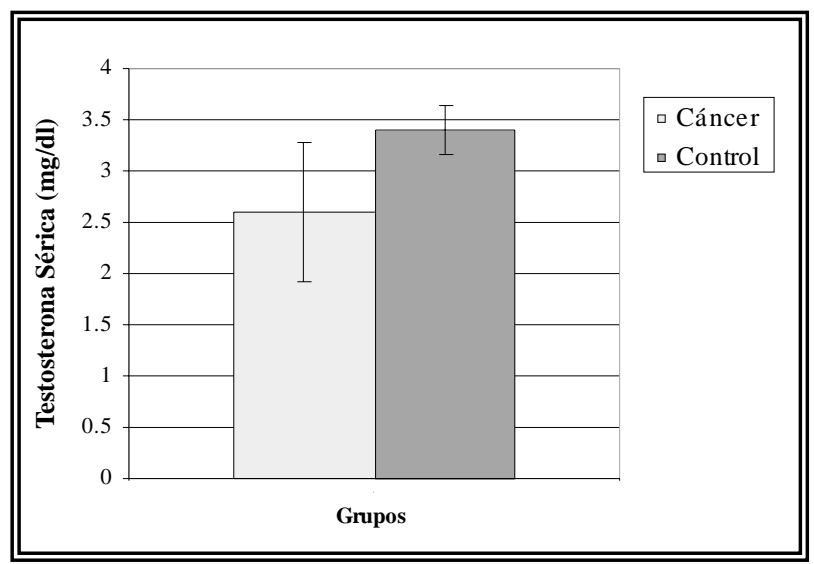

FIGURA 1. Testosterona total $(\mathrm{ng} / \mathrm{ml})$ en pacientes con cáncer versus control (intervalo de confianza $95 \%$ ).

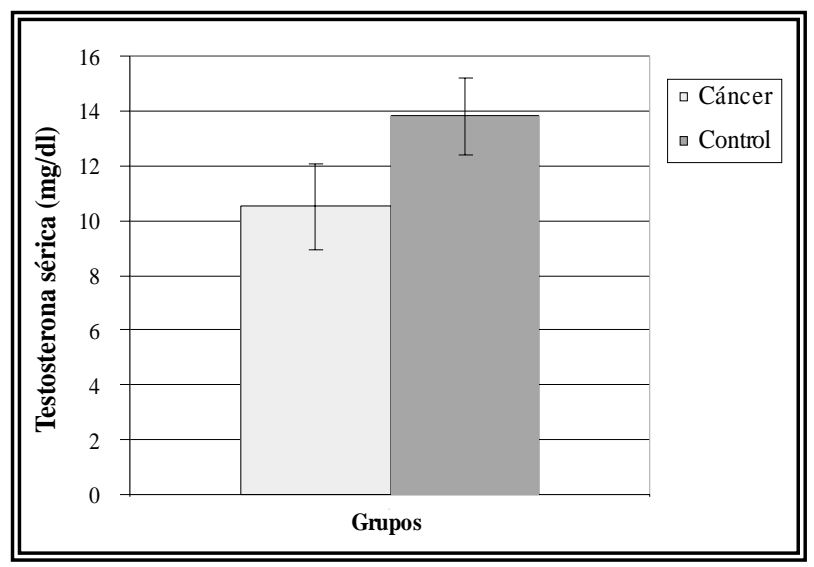

FIGURA 2. Testosterona libre $(\mathrm{pg} / \mathrm{ml})$ en pacientes con cáncer versus control (intervalo de confianza 95\%)
TABLA V

RELACIÓN GLEASON Y PROMEDIO
DE TESTOSTERONA LIBRE
\begin{tabular}{|c|c|}
\hline Gleason & $\begin{array}{c}\text { Testosterona libre (promedio) } \\
\mathrm{pg} / \mathrm{ml}\end{array}$ \\
\hline 5 & 9,8 \\
\hline 6 & 11,48 \\
\hline 7 & 9,21 \\
\hline$\geq 8$ & 10,37 \\
\hline
\end{tabular}

El promedio de APE fue de 71,17 ng/ml en los pacientes con cáncer y $1,18 \mathrm{ng} / \mathrm{ml}$ en el grupo control, tampoco hubo relación con testosterona.

La etapificación demostró un $36,8 \%$ en etapas T1 y $\mathrm{T} 2$ con un $78,5 \%$ de prostatectomía radical. El resto fueron etapas avanzadas (Tabla VI). No constatamos ninguna relación entre las distintas etapas y la disminución de la testosterona.

TABLA VI

ETAPIFICACIÓN PACIENTES CON CÁNCER SEGÚN TRATAMIENTO

\begin{tabular}{|c|c|c|}
\hline Etapa Clínica & Hormonoterapia & Prostatectomía \\
\hline T1 & & 4 \\
\hline T2 & 3 & 7 \\
\hline T3 & 13 & 4 \\
\hline T4 & 7 & 0 \\
\hline Total & $\mathbf{2 3}$ & $\mathbf{1 5}$ \\
\hline
\end{tabular}

\section{DISCUSIÓN}

La sintesis de testosterona es requerida para los caracteres sexuales secundarios y conducta sexual normal. Esta es regulada por el lóbulo anterior de la hipófisis mediante la liberación de hormona luteinizante (LH) y ésta a su vez por el hipotálamo con la liberación de hormona liberadora de gonadotrofina (GHRH) instalándose entre ellos un feedback directo.

La LH tiene afinidad muy directa con los receptores de membrana plasmática de las células de leydig, lo que estimula a través de la adenylato ciclasa el aumento de la formación de cAMP en el proceso bioquímico para llegar a la testosterona, (pasando por colesterol, pregnenolona, progesterona, 17 hidroxiprogesterona, androstenediona). Esta testosterona se puede dirigir a los túbulos seminíferos o a los tejidos 
periféricos androgénicos en forma de testosterona, dihidrotestosterona o estradiol (si es aromatisada)

La producción de testosterona en el hombre es en forma pulsátil con un peak en la mañana y nadir en la tarde. Existe en un $2 \%$ en forma libre, un $30 \%$ unida a la globulina sexual con gran afinidad (no es biológicamente activa), y el resto se une a la albúmina. La testosterona libre y esta última es la llamada testosterona biodisponible.

Sabemos que la medición de testosterona total no es representativa de lo que realmente ocurre en el organismo y que la libre es muy superior, pero los verdaderos datos exactos son revelados con la medición de la testosterona biodisponible.

Los andrógenos son las principales hormonas reguladoras del crecimiento y desarrollo normal de la próstata. El uso de la deprivación de los andrógenos está basado en el trabajo de Huggins que demostró la dependencia del cáncer a éstos ${ }^{4}$. Altas dosis de estrógenos son usados exitosamente en el tratamiento del cáncer prostático llevando los valores de la testosterona del suero a niveles de castración ${ }^{5,6}$.

Según los trabajos de Ho, la administración simultánea de estrógenos y testosterona en ratas induce aumento de proliferación epitelial, mitosis y frecuencia de carcinoma. Andrógenos o estrógenos por sí solos, no producen estos cambios ${ }^{7}$.

Los eunucos o pacientes orquiectomizados no hacen cáncer prostático. Se supone entonces, que a un aumento importante de la testosterona, aumenta también el estradiol por aromatización y si el individuo es obeso, las posibilidades de acumular estradiol son mayores, por tanto, la posibilidad de hacer cáncer prostático también es mayor, por tener mayores niveles de testosterona y estrógenos.

Carlstrom demostró en un estudio de 72 pacientes con cáncer prostático un aumento de la testosterona total y la biodisponible, en relación al grupo control, además demostró un aumento de los estrógenos periféricos producto de aromatización ${ }^{8}$, lo que concuerda con el trabajo de $\mathrm{Ho}^{7}$ como probable etiología de cáncer prostático.

En una revisión de 23 trabajos de casos y controles presentada por Anderson en 1993, demostró que a pesar de que el cáncer de próstata es un tumor andrógeno dependiente, los valores de tes- tosterona fueron normales o bajos en 19 de los 23 estudios $^{9}$, similares hechos fueron también reportados para los niveles de estrógenos ${ }^{10}$.

Robinson y Thomas demostraron que pacientes con cáncer prostático metastásico tenían niveles de testosterona más bajos que aquellos sin metástasis ${ }^{11}$. Harper estudió 222 pacientes y encontró que los fallecidos antes de 1 año de tratamiento tenían testosterona significativamente más baja que los que sobrevivían al año ${ }^{12}$. Wilson demostró que los pacientes con niveles de testosterona en suero mayores a $200 \mathrm{ng} / \mathrm{ml}$ tenían mejor pronóstico ${ }^{13}$.

La explicación de estos resultados estaría en un trabajo de Miller, quien demostró un aumento significativo de los niveles en suero de testosterona total y libre, estradiol, LH y FSH y una disminución significativa de dihidrotestosterona posterior a un año de la prostatectomía radical ${ }^{14}$, lo cual, significaría que la próstata puede producir substancias que suprimen los niveles de estas hormonas en el suero.

Se sabe que la próstata, al igual que el testículo producen inhibina, que es una glicoproteína, la cual inhibe la producción de gonadotrofinas, especialmente la $\mathrm{FSH}^{15}$. De acuerdo a lo anterior, supondríamos que el cáncer prostático es favorecido en su iniciación por los andrógenos; pero una vez instalado, éste haría bajar la testosterona.

Schatzi ${ }^{16}$ demostró recientemente que los bajos niveles de testosterona y estradiol en el suero de pacientes con cáncer prostático, se asociaban directamente con el mayor grado de Gleason.

\section{CONCLUSIONES}

Nuestro trabajo es concordante con la mayoría de los publicados, pero no hubo correlación con etapa tumoral ni Gleason.

\section{REFERENCIAS}

1. CARTER HB, PEARSON JD, METTER EJ et al.: Longitudinal evaluation of serum androgen levels in men with and without prostate cancer. Prostate 1995; 25: 27.

2. MORGENTALER A, BRUNING CO, III DEWOLF WC.: Occult prostate cancer in men with low serum testosterone levels. JAMA 1996; 276: 1904.

3. HOFFMAN M, DEWOLF W, MORGENTALER A.: Is low serum free testosterone a marker for high grade prostate cancer? J Urol 2000; 163: 824-827. 
4. HUGGINS C, STEVENS R, HODGES C.: Studies en prostatic cancer. The effects of castration on advanced carcinoma of the prostate gland. Arch Surg 1941; 43: 209.

5. STEGE R, CARLSTROM K, COLLSTE L et al.: Single drug parenteral estrogen treatment in prostatic cancer. The Prostate 1989; 14: 183-188.

6. HENRIKSSON P, BLOMBACK M, ERIKSSON A, STEGE R, CARLSTROM K.: Effects of parenteral oestrogen on the coagulation system in patients with prostatic carcinoma. Br J Urol 1990; 65: 282-285.

7. HO SM, YU M, LEAV I, VICCIONE T.: The conjoint action of androgens and oestrogens in the induction of proliferative lesions in the prostate. In $\mathrm{Li} \mathrm{jj}$ Noandi SA eds. Hormonal Carcinogenesis. New York: Sringer-Verlag 1992; 18-25.

8. CARLSTROM K, STEGE R.: Testicular and adrenocortical function in men with prostatic cancer and in healthy age-matched controls. $\mathrm{Br} J$ Urol 1997; 79: $427-431$

9. ANDERSON SO, ADAMI HO, BERGSTROM R, WIDW L.: Serum pituitary and sex steroid hormone levels in the aetiology of prostatic cancer. $\mathrm{Br} J$ Cancer 1993; 68: 97-102.

10. FEHER T, KOREF O, SZENDROI Z, CSELLAR M.: Androgen metabolites in blood of patients with prostatic carcinoma. Int Urol Nephrol 1970; 2: 163169.

11. ROBINSON M, THOMAS B.: Effect of hormonal therapy on plasma testosterone levels in prostatic carcinoma. Brit Med J 1971; 4: 391.
12. HARPER M, PIERREPOINT C, GRIFFITH K.: Carcinoma of the prostate: relationship of pretreatment hormone levels to survival. Eur J Cancer Clin Oncol 1984; 20: 477.

13. WILSON D, HARPER M, JENSEN H, IKEDA R, RICHARDS G, PEELING W, PIERREPOINT C, GRIFFITHS K.: A prognostic index for the clinical management of patients with advanced prostatic cancer. Prostate 1985; 7: 131.

14. MILLER L, PARTIN A, CHAN D, BRUZEK D, DOBS A, EPSTEIN J, WALSH P.: Influence of radical prostatectomy on serum hormone levels. J Urol 1998; 160: 449-453.

15. PHADKE M, VANAGE G, SHETH A.: Circulating levels of inhibin, prolactin, TSH, Lh and FSH in benign prostatic hypertrophy before and after tumor resection. Prostate 1987; 10: 115.

16. SCHATZI G, MADERSBACHER S, THURRIDI T, WALDMÜLLER J, KRAMER G, HAITEL A, MARBERGER M.: High-grade prostate cancer is associated with low serum testosterone levels. Prostate 2001; 47: 52-58.

Dr. Pedro Rivera Garay

Trizano, 150

Temuco (Chile)

(Trabajo recibido el 20 febrero de 2003) 